\title{
Correlates of NNAL levels among nondaily and daily smokers in the college student population
}

\author{
This article was published in the following Dove Press journal: \\ Current Biomarker Findings \\ 15 October 2012 \\ Number of times this article has been viewed
}

\section{Carla J Berg' \\ Gillian L Schauer' \\ Jasjit S Ahluwalia ${ }^{2}$ \\ Neal L Benowitz ${ }^{3}$ \\ 'Department of Behavioral Sciences and Health Education, Emory \\ University School of Public Health, Atlanta, GA, ${ }^{2}$ Department of Medicine and Center for Health Equity, University of Minnesota, Minneapolis, $\mathrm{MN},{ }^{3}$ Departments of Medicine and Bioengineering and Therapeutic Sciences, University of California, San Francisco, CA, USA}

Correspondence: Carla J Berg

Department of Behavioral Sciences and Health Education, Emory University,

I5I 8 Clifton Road NE, 5th Floor,

Atlanta, GA 30322, USA

Tel +l 4047277589

Fax + I 404727 I 369

Email cjberg@emory.edu
Introduction: Recent simultaneous increases in nondaily smoking and decreases in daily smoking make the identification of nondaily smokers through biomarker measures as well as the relationship of biomarker levels to smoking behaviors important topics. However, little is known about biochemical identification and carcinogen exposure of nondaily smokers. One tobacco-specific nitrosamine, 4-(methylnitrosamino)-1-(3-pyridyl)-1-butanol (NNAL), has a long half-life, making it a useful marker for long-term and intermittent tobacco exposure. Thus, we examined correlates of urine NNAL levels among nondaily and daily smokers.

Methods: In 2011, we obtained urine samples from 64 current cigarette smokers (37 nondaily; 27 daily) in the Southeastern US and assessed participants' sociodemographics, smoking-related information, and other tobacco use. Our sample included 14 participants concurrently using other combustible tobacco products and eight concurrently using smokeless tobacco.

Results: Of six participants smoking for only one day in the past 30, four had detectable NNAL levels; thus, two nondaily smokers were excluded from the remainder of the analyses. In multivariate analysis, average cigarettes per day on smoking days $(B=23.00,95 \%$ Confidence Interval $[\mathrm{CI}] 13.81,32.20, P<0.001)$ and number of days of smokeless tobacco use $(\mathrm{B}=17.11$, CI 13.53, 20.70, $P<0.001)$ were associated with NNAL levels among nondaily smokers $\left(\mathrm{R}^{2}=0.234\right)$. Multivariate analysis indicated that average cigarettes per day $(\mathrm{B}=15.83, \mathrm{CI} 2.89$, $28.76, P=0.02$ ) was the only significant correlate of NNAL levels among daily smokers. We used receiver operating characteristic (ROC) analyses to identify a potential urinary NNAL (normalized for creatinine) cutoff point of $81.6 \mathrm{pg} / \mathrm{mL} / \mathrm{g}$ creatinine $(88.9 \%$ sensitivity, $80.0 \%$ specificity) to discriminate nondaily from daily smokers. Excluding polytobacco-product users from these analyses provided similar results.

Conclusion: Different correlates of NNAL levels exist among nondaily and daily cigarette smokers. Urine NNAL demonstrates the potential to be used to discriminate nondaily from daily smokers among young adults.

Keywords: biomarkers, smoking, carcinogen, tobacco

\section{Introduction}

Smoking remains the leading causes of preventable disease in the US. ${ }^{1}$ Despite efforts to decrease its prevalence, $20.6 \%$ of American adults continue to smoke. ${ }^{2}$ While daily smoking is declining, ${ }^{3,4}$ nondaily smoking (smoking on some days but not every day) is increasing. ${ }^{5}$ Estimates from the 2008 National Survey on Drug Use and Health ${ }^{6}$ and from the 2006 Behavioral Risk Factor Surveillance Survey data ${ }^{7}$ indicate that between a fourth and a third of adults report nondaily smoking. Young adults have been particularly affected by the increase in nondaily smoking. ${ }^{8}$ 
While nondaily smoking may be a transitory condition between daily smoking and quitting ${ }^{9-11}$ or a transitional phase to heavier or regular cigarette use, ${ }^{12}$ some research shows that this pattern of smoking may continue indefinitely. ${ }^{13-15}$ While nondaily smokers can abstain from tobacco for days without exhibiting signs of withdrawal, ${ }^{16}$ other findings indicate that they may experience urges to smoke and difficulty achieving cessation as a result of physiological addiction..$^{16,17}$ Unfortunately, nondaily smokers suffer from significant smoking-related morbidity and mortality compared with never-smokers. ${ }^{18-20}$ Thus, this is an important pattern of smoking to not only identify through self-report measures but to also confirm through the use of biomarker assessment. Moreover, it is important to understand how self-reported smoking behaviors may be associated with tobacco-related biomarker levels among nondaily smokers.

Biomarkers can be useful in assessing smokers' exposure to tobacco smoke. Self-reported smoking patterns such as number of days smoked and cigarettes smoked per day are imprecise measures of smoke exposure, particularly among nondaily smokers. Little research has been published to identify the best self-reported factors associated with carcinogen exposure among different groups of smokers (ie, nondaily smokers, daily smokers, smokers using other combustible tobacco products). Prior research has suggested that exposure patterns differ significantly from person to person due to differences in how cigarettes are smoked, differences in tobacco products, and several other factors..$^{21}$ Furthermore, prior research has not identified an optimal biomarker cutoff point for distinguishing nondaily smokers from daily smokers.

Two biomarkers are commonly used to identify tobacco smoke exposure: cotinine and 4-(methylnitrosamino)-1(3-pyridyl)-1-butanol (NNAL). Cotinine, the major proximate metabolite of nicotine, is widely used to distinguish smokers from nonsmokers in epidemiological studies and smoking cessation trials. ${ }^{22-24} \mathrm{~A}$ limitation of the use of cotinine is its relatively short half-life (16 hours), making its interpretation particularly problematic with nondaily smoking. Tobacco-specific nitrosamines are formed from nicotine and other tobacco alkaloids during tobacco curing and burning, and are carcinogens. ${ }^{25}$ NNAL is a metabolite of 4-(methylnitrosamino)-1-(3-pyridyl)1-butanone (NNK), a potent lung carcinogen, and its levels are associated with lung cancer. ${ }^{26}$ The half-life of NNAL (10-16 days) is much longer than that of cotinine, suggesting that NNAL might be a better measure of tobacco exposure over time or in cases where exposure is highly variable. This is especially relevant for nondaily smokers whose smoking patterns may vary over time. ${ }^{27}$
Given the aforementioned research, our study aimed to examine urine NNAL levels and correlates of NNAL among nondaily smokers and daily smokers in a college student population. This information may provide data regarding how to distinguish nondaily from daily smokers, which may have great utility in examining smoking patterns and changes among young adult smokers.

\section{Methods}

\section{Participants and procedures}

In Spring 2011, we conducted 16 in-person focus groups with 64 college-student, past 30-day smokers (27 daily smokers, 37 nondaily smokers) drawn from three colleges (two technical colleges, one university) in the Southeastern US. Participants were recruited from an online survey conducted in Fall 2010 (see Berg ${ }^{28}$ for survey administration details) to qualitatively examine their smoking history, patterns, and attitudes toward smoking and cessation. Participants were screened and recruited into one of the focus groups, with each group being homogenous for gender, school type, and smoking status (nondaily vs daily smoker). The current study examines biomarker data collected during these in-person meetings. The Emory University Institutional Review Board approved this study, IRB\# 00030631.

\section{Measures}

Prior to the group discussion, participants completed the informed statement of consent, provided urine for biomarker measurement, and completed a brief survey assessing sociodemographics (age, gender, and ethnicity), body mass index (BMI), smoking behaviors, and other tobacco use. In terms of tobacco-related assessments, we asked, "In the past 30 days, on how many days did you smoke a cigarette (even a puff)?" ${ }^{29,30}$ Those reporting smoking on all 30 days were considered daily smokers; those smoking between 1 and 29 days in the past 30 days were considered nondaily smokers. ${ }^{31,32}$ Participants also completed a seven-day timeline, retrospectively reporting the number of cigarettes smoked each day. This was aggregated into a variable indicating the total number of cigarettes in the past week. We also asked, "Do you typically smoke menthol cigarettes?" and "How soon after you first wake do you smoke your first cigarette?" with response options of: within 5 minutes, 6-30 minutes, 31-60 minutes, or after 60 minutes. ${ }^{33}$ This variable was dichotomized as within 30 minutes versus after 30 minutes. ${ }^{33}$ We also asked, "In the past 30 days, on how many days did you use some other form of combustible tobacco, such as cigars or other 
smoking tobacco products (not including cigarettes)? Use smokeless tobacco?" 29,30

\section{Data analysis}

NNAL was analyzed by liquid chromatography mass spectrometry as described by Jacob et $\mathrm{al}^{34}$ and was normalized for urine creatinine. The limit of quantification was $0.25 \mathrm{pg} / \mathrm{mL}$. Descriptive statistics were presented using means and standard deviations for continuous variables and frequency and percentage for categorical variables. We conducted bivariate analyses examining differences in smoking behaviors and NNAL between nondaily and daily smokers. We then examined correlations among sociodemographics and smoking-related characteristics in relation to NNAL among nondaily smokers and daily smokers, respectively. We then excluded users of other tobacco products (both combustible and smokeless) and reran these analyses to isolate the specific relationships of these factors to NNAL levels specifically among cigarette users. Next, we developed multivariate models to identify correlates of NNAL among nondaily and daily smokers, respectively. To estimate potential NNAL cutoff points that would distinguish nondaily from daily smokers, we generated receiver operating characteristic (ROC) curves and assumed that both sensitivity and selectivity had the same importance. This was conducted using NNAL both with and without normalization for creatinine, and among all participants as well as among those not using noncigarette tobacco products. Quantitative data were analyzed using PASW 19.0 (IBM, New York, NY).

\section{Results}

Table 1 presents data on means of NNAL among nondaily and daily smokers. Of the six participants reporting only 1 day of smoking in the past 30 days, four participants had detectable levels. Of these six participants, only one participant used other tobacco products (ie, one day of other combustible tobacco product use) and had detectable NNAL levels. The two nondaily smokers who had undetectable NNAL levels were excluded from the remainder of the analyses. Figure 1 displays a scatterplot indicating number of days of smoking in the past 30 days relative to NNAL levels.

Table 1 shows that use of noncigarette tobacco products was more prevalent among nondaily smokers than daily smokers. We found that among nondaily smokers, number of days of smoking was associated with cigarettes smoked per day (cpd) $(\mathrm{r}=0.65, P<0.001)$, number of days of smokeless tobacco use $(\mathrm{r}=0.39, P=0.02)$, and number of other combustible tobacco products used $(\mathrm{r}=0.35, P=0.03$; not included in Table 1). However, cpd among both nondaily and daily smokers was unrelated to number of days of smokeless tobacco or other combustible tobacco use. Among nondaily smokers, there was also a correlation between number of days using smokeless tobacco and other combustible tobacco products $(\mathrm{r}=0.55, P<0.001)$, but this relationship was not found among daily smokers.

Table 1 also displays correlates of NNAL normalized for creatinine among nondaily and daily smokers. Among nondaily smokers, NNAL levels were significantly associated with number of days of smoking in the past month, average cpd on smoking days, use of smokeless tobacco in the past 30 days, number of days of smokeless tobacco use in the past month, and use of other combustible tobacco products in the past 30 days. Among daily smokers, NNAL levels were associated with age, cpd, number of cigarettes smoked in the past week, and smoking within 30 minutes of waking. We also conducted the same analyses excluding all users of other tobacco products (both combustible and smokeless), and results were similar.

In multivariate analysis examining correlates of NNAL among nondaily smokers, average cpd on smoking days ( $\mathrm{B}=23.0,95 \%$ Confidence Interval $[\mathrm{CI}] 13.8,32.2, P<0.001)$ and number of days of smokeless tobacco use $(\mathrm{B}=17.1$, CI 13.5, 20.7, $P<0.001$ ) were associated with NNAL levels $\left(\mathrm{R}^{2}=0.234\right)$. Excluding other tobacco (combustible and smokeless) users, the only significant correlate was average cpd on smoking days ( $\mathrm{B}=8.1, \mathrm{CI} 2.7,13.5, P=0.006$ ). Among daily smokers, multivariate analysis indicated that $\mathrm{cpd}$ ( $\mathrm{B}=15.8, \mathrm{CI} 2.9,28.8, P=0.02$ ) was the only significant correlate of NNAL levels. Excluding other tobacco (combustible and smokeless) users resulted in similar results.

To examine potential cutoff points for determining nondaily versus daily smoking status in our sample of current smokers, we conducted ROC analyses (see Figure 2). Using NNAL not normalized for creatinine among all participants, ROC indicated $84.9 \%$ area under the curve $(P<0.001)$. The optimal cutoff point of NNAL to discriminate nondaily versus daily smokers was $75.9 \mathrm{pg} / \mathrm{mL}$ (81.5\% sensitivity, 83.9\% specificity). Using NNAL normalized for creatinine, ROC indicated $87.6 \%$ area under the curve $(P<0.001)$. The optimal cutoff point of NNAL normalized for creatinine to discriminate nondaily versus daily smokers was $81.6 \mathrm{pg} / \mathrm{mL} / \mathrm{g}$ creatinine ( $88.9 \%$ sensitivity, $80.0 \%$ specificity).

We then excluded those reporting the use of other tobacco products (combustible and smokeless). Using NNAL not normalized for creatinine, ROC indicated $95.3 \%$ area under the curve $(P<0.001)$. The optimal cutoff point of NNAL to 


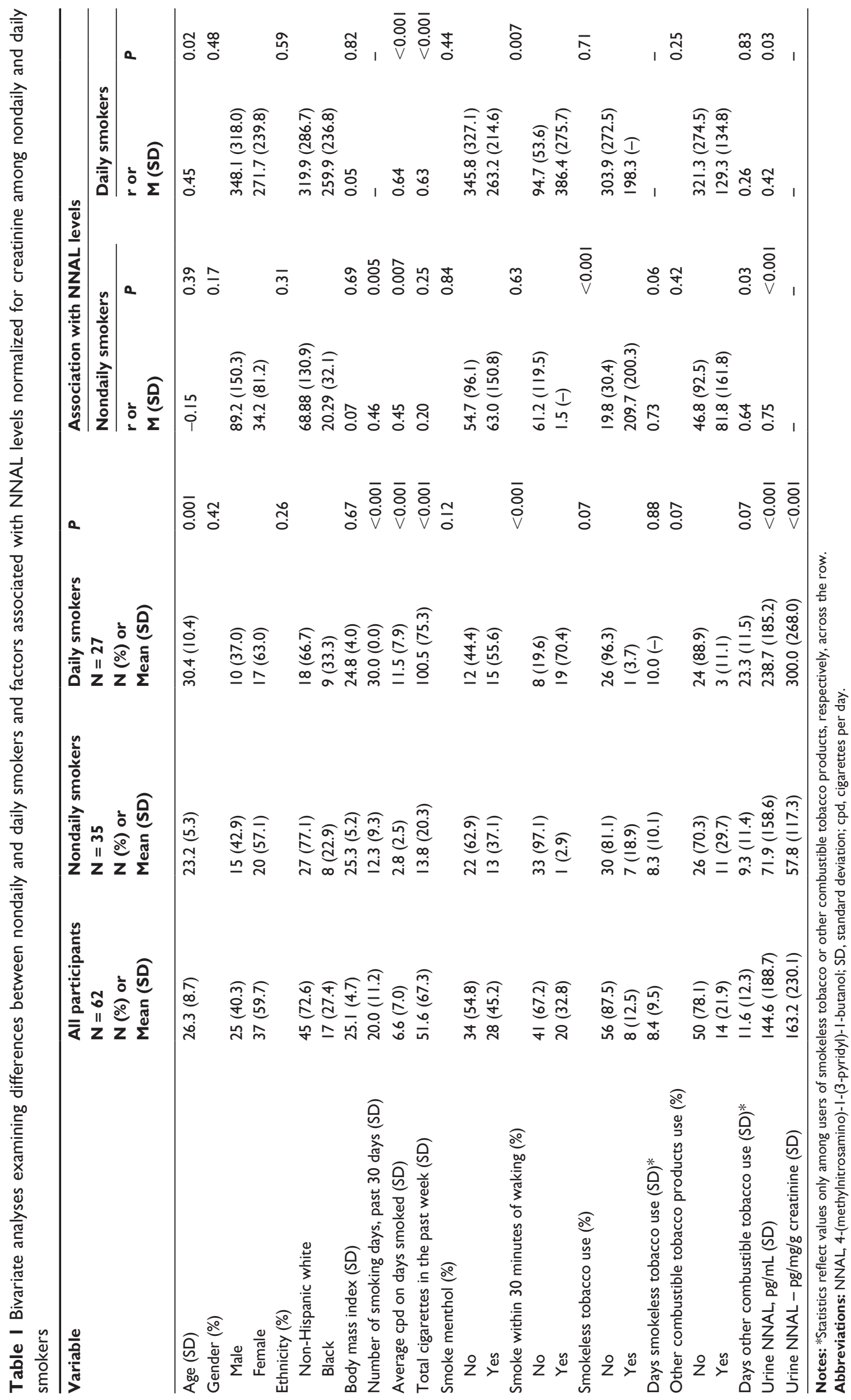




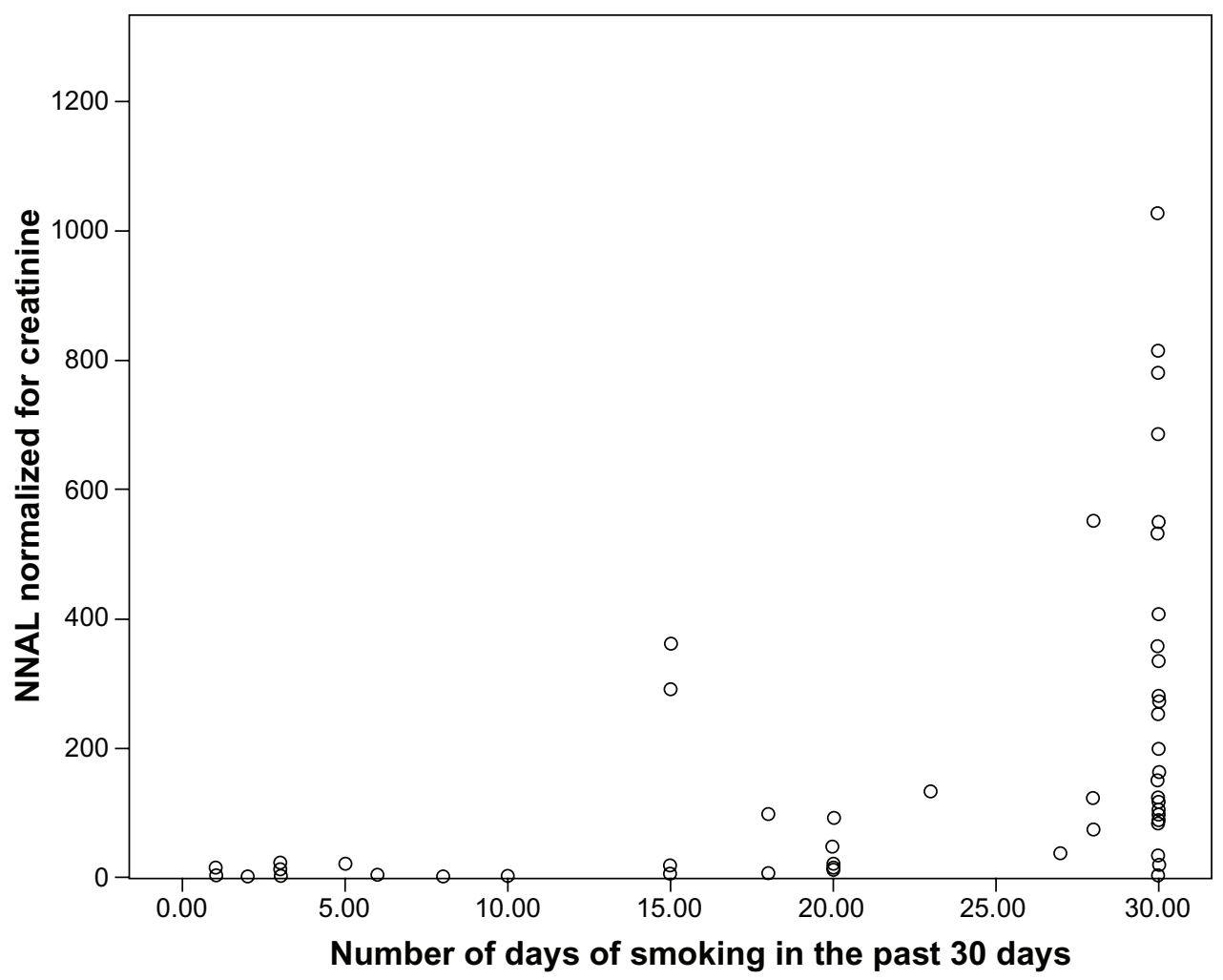

Figure I Scatterplot of smoking frequency in the past month and NNAL levels normalized for creatinine (pg/mL/g creatinine). Abbreviation: NNAL, 4-(methylnitrosamino)-I-(3-pyridyl)-I-butanol.

discriminate nondaily versus daily smokers was $50.2 \mathrm{pg} / \mathrm{mL}$ (91.3\% sensitivity, 90.9\% specificity). Examining NNAL normalized for creatinine, ROC indicated $97.0 \%$ area under the curve $(P<0.001)$. The optimal cutoff point of NNAL to discriminate nondaily versus daily smokers was $70.5 \mathrm{pg} / \mathrm{mL} / \mathrm{g}$ creatinine (91.3\% sensitivity, 95.5\% specificity).

\section{Discussion}

This study describes urine NNAL levels and unique correlates of NNAL among nondaily and daily smokers. Importantly, we found that of the six participants reporting only 1 day of smoking in the past 30 days, four participants had detectable levels. Thus, even very low levels of smoking were detectable, and NNAL was a useful way to detect past 30-day intermittent smoking. Findings also suggest that the factors related to NNAL levels among nondaily and daily smokers were different.

Among nondaily smokers, NNAL levels were associated with number of days of smoking in the past month, average cpd on smoking days, and use of other tobacco use, both combustible and smokeless. Multivariate analyses indicated that cpd on smoking days and frequency of smokeless tobacco use were associated with NNAL levels. Interestingly, number of days of smoking was not the most significant correlate.
This may be due to the high correlation between smoking frequency and cpd among nondaily smokers. Moreover, the high correlation between use of smokeless and combustible tobacco products may account for smokeless tobacco being more significantly associated with NNAL levels in multivariate analyses. Excluding noncigarette users indicated cpd to be the major correlate of NNAL. Another interesting finding is that NNAL levels were associated with average cpd but not cigarette consumption in the past week. Given the long half-life of NNAL, this may be due to low levels of recent cigarette consumption among some nondaily smokers but potentially greater consumption over the past 30 days.

Among daily smokers, NNAL levels were associated with age, cpd, cigarettes smoked in the past week, and smoking within 30 minutes of waking. Smoking level and nicotine dependence are previously documented correlates of NNAL levels in regular smokers. ${ }^{27}$ Among daily smokers, smokeless tobacco use and other combustible tobacco use were not associated with NNAL levels. This is most likely due to the low levels of alternative tobacco use and the already high level of cigarette consumption among daily smokers.

We determined an optimal cutoff point of $75.9 \mathrm{ng} / \mathrm{mL}$ for urine NNAL with $81.5 \%$ sensitivity and $83.9 \%$ specificity to distinguish nondaily versus daily smokers. Prior research 
A

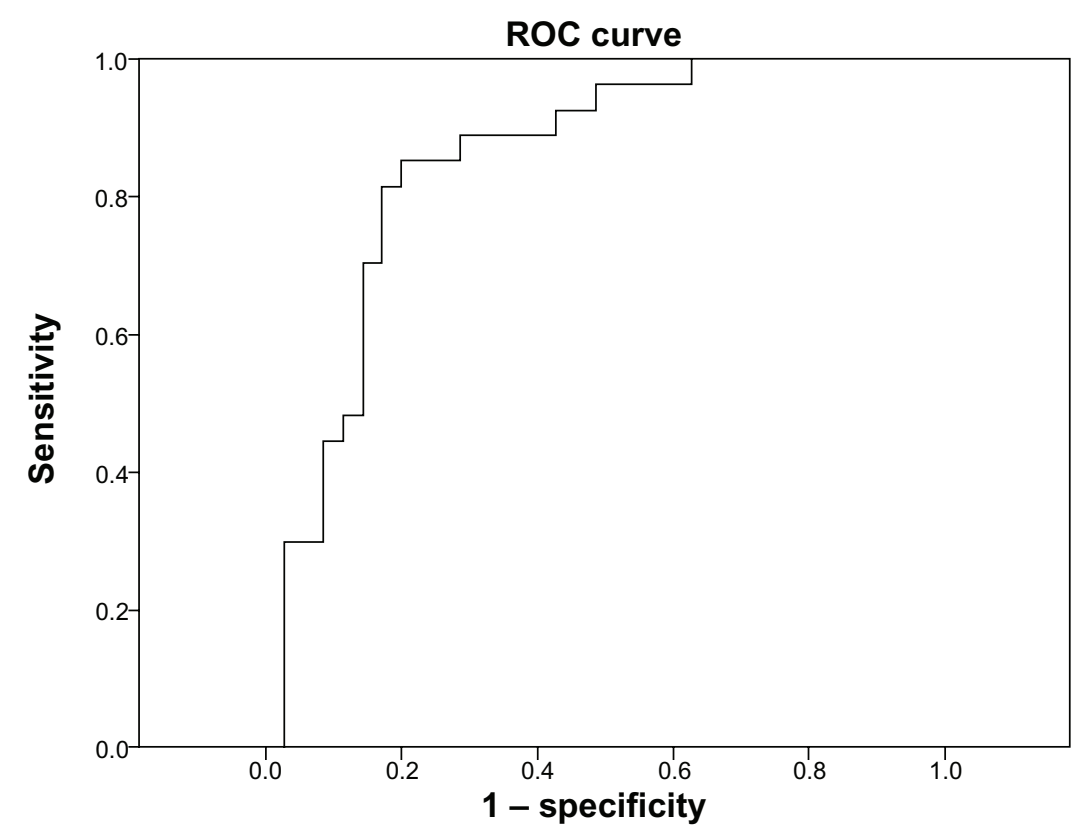

B

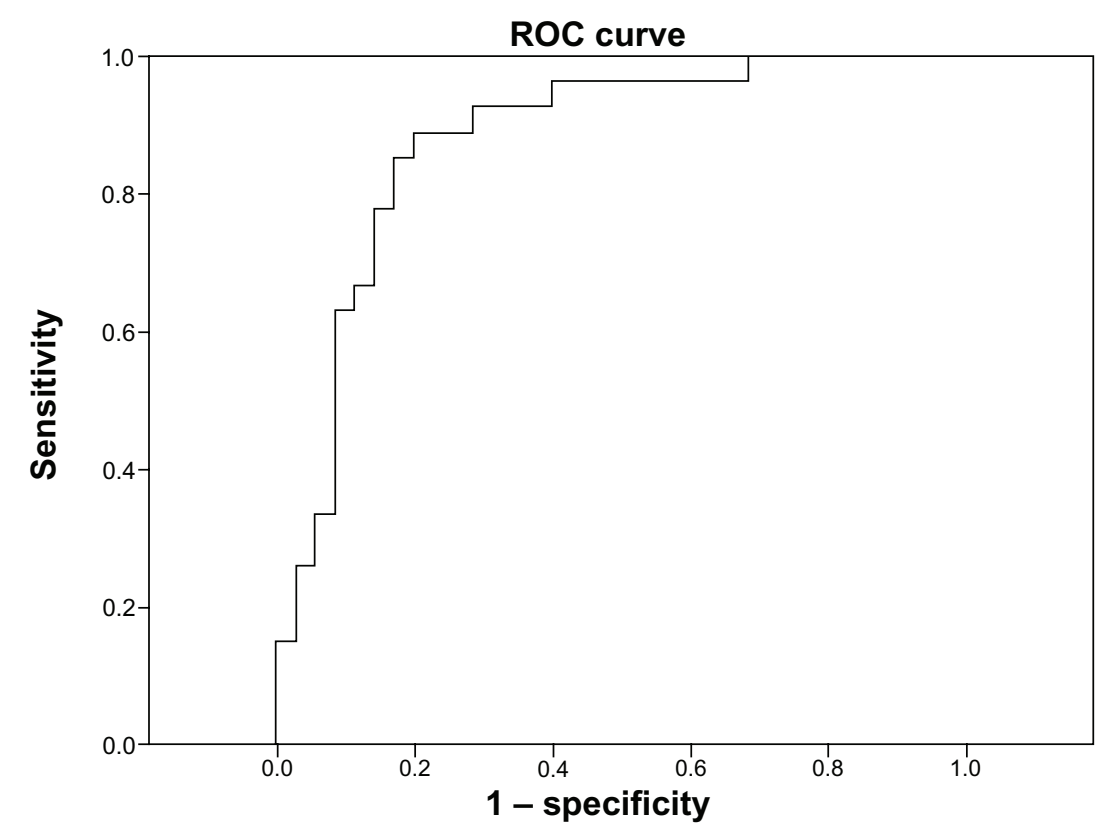

Figure 2 Validity of proposed optimal cutoff in predicting smoking status for NNAL (A) and NNAL normalized for creatinine (B).

Abbreviations: NNAL, 4-(methylnitrosamino)-I-(3-pyridyl)-I-butanol; ROC, receiver operating characteristic.

by Goniewicz et $\mathrm{al}^{35}$ on 373 active smokers and 228 passive smokers indicated an optimal cutoff point of $47.3 \mathrm{pg} / \mathrm{mL}$ to determine active versus passive smoking, which had specificity of $96.5 \%$ and sensitivity of $87.4 \%$. The Goniewicz study included nondaily smokers within the smoker group, so our present estimates comparing nondaily versus daily smokers are consistent with expectations. ${ }^{35}$ As expected, excluding polytobacco users resulted in a lower optimal cutoff point ( $50.2 \mathrm{pg} / \mathrm{mL}$ with higher sensitivity and specificity). This is an important finding, indicating that nondaily smokers who are not polytobacco users demonstrated similar NNAL levels to passive smokers in the Goniewicz study. Taken together, these potential cutoff points may inform future clinical trials aiming to identify eligible smokers, depending on the 
smoker population (ie, either daily or nondaily) targeted by the intervention. Moreover, it may help to inform our assessments of the success of interventions targeting nondaily smokers, as we might be able to identify whether nondaily smokers transition to a level of cigarette consumption and carcinogen exposure similar to daily smokers.

\section{Limitations}

Limitations to the current study include small sample size, limited generalizability, the use of self-reported smoking behaviors, the inclusion of polytobacco users, and a lack of secondhand smoke-exposure assessment (which may have also impacted NNAL levels). Also, our dichotomization of daily (all 30 days in the past 30 days) versus nondaily ( $<30$ days in the past 30 days) is a concern; however, none of the nondaily smokers smoked more than 25 days of the past 30 . Finally, some of the associations documented were weak, which may have also been due to the small sample size.

\section{Conclusion}

Despite these limitations, this study provided important results and has implications for research. First, we provide novel information on the correlates of urine NNAL levels among nondaily and daily smokers in the young adult population. Second, we present potential urine NNAL cutoff points to discriminate nondaily versus daily smokers, which may be useful given the ability of urine NNAL to be used when subjects have not smoked for some period of time or have only smoked on a few days of the past month. Given our small sample size, our findings serve as a basis for methodological replication with a larger sample to establish a cutoff point between daily and nondaily smokers in terms of urine NNAL. Finally, there were few differences in our findings despite inclusion of users of other tobacco products, except in terms of predicting NNAL levels among nondaily smokers. Given the high rate of alternative tobacco product use and polytobacco use among nondaily smokers in the young adult population, the current findings have real world applicability.

\section{Acknowledgments}

This research was supported by the National Cancer Institute (1K07CA139114-01A1; PI: Berg) and the Georgia Cancer Coalition (PI: Berg). Dr Ahluwalia is supported in part by 1P60MD003422 from the National Institute for Minority Health and Health Disparities. Dr Benowitz has instrumentation and analytical chemistry support from the National Institutes of Health grants S10 RR026437 and P30 DA01293.
NL Benowitz received support from the Flight Attendants Medical Research Institute. We would like to thank our collaborators across the state of Georgia for their assistance in conducting focus groups and collecting data.

\section{Disclosure}

The authors declare no conflicts of interest.

\section{References}

1. US Department of Health and Human Services. Healthy People 2010: With Understanding and Improving Health and Objectives for Improving Health. 2nd ed. Washington: US Government Printing Office; 2000.

2. Centers for Disease Control and Prevention. Smoking and Tobacco Use. Atlanta: Centers for Disease Control and Prevention; 2011.

3. Pierce JP, White MM, Messer K. Changing age-specific patterns of cigarette consumption in the United States, 1992-2002: association with smoke-free homes and state-level tobacco control activity. Nicotine Tob Res. 2009;11(2):171-177.

4. Centers for Disease Control and Prevention. Behavioral Risk Factor Surveillance System Survey Data. Atlanta: US Department of Health and Human Services, Centers for Disease Control and Prevention; 2010.

5. Centers for Disease Control and Prevention. Prevalence of current cigarette smoking among adults and changes in prevalence of current and some day smoking - United States, 1996-2001. MMWR Morb Mortal Wkly Rep. 2003;52(14):303-304, 306-307.

6. Substance Abuse and Mental Health Services Administration. Results from the 2008 National Survey on Drug Use and Health: National findings. Rockville: Office of Applied Studies; 2009. Available from: http://www.samhsa.gov/data/nsduh/2k8nsduh/2k8Results.htm. Accessed September 19, 2012.

7. Centers for Disease Control and Prevention. Cigarette smoking among adults - United States, 2006. MMWR Morb Mortal Wkly Rep. 2007;56(44):1157-1161.

8. Schane RE, Glantz SA, Ling PM. Nondaily and social smoking: an increasingly prevalent pattern. Arch Intern Med. 2009;169(19): $1742-1744$.

9. Evans NJ, Gilpin E, Pierce JP, et al. Occasional smoking among adults: evidence from the California tobacco survey. Tob Control. 1992;1: 169-175.

10. Okuyemi KS, Harris KJ, Scheibmeir M, Choi WS, Powell J, Ahluwalia JS. Light smokers: issues and recommendations. Nicotine Tob Res. 2002; 4 Supp1 2:S103-S112.

11. Zhu SH, Sun J, Hawkins S, Pierce J, Cummins S. A population study of low-rate smokers: quitting history and instability over time. Health Psychol. 2003;22(3):245-252.

12. White HR, Bray BC, Fleming CB, Catalano RF. Transitions into and out of light and intermittent smoking during emerging adulthood. Nicotine Tob Res. 2009;11(2):211-219.

13. Hassmiller KM, Warner KE, Mendez D, Levy DT, Romano E. Nondaily smokers: who are they? Am J Public Health. 2003;93(8):1321-1327.

14. Shiffman S. Light and intermittent smokers: background and perspective. Nicotine Tob Res. 2009;11(2):122-125.

15. Hennrikus DJ, Jeffery RW, Lando HA. Occasional smoking in a Minnesota working population. Am J Public Health. 1996;86(9): $1260-1266$.

16. Rubinstein ML, Benowitz NL, Auerback GM, Moscicki AB. Withdrawal in adolescent light smokers following 24-hour abstinence. Nicotine Tob Res. 2009;11(2):185-189.

17. DiFranza JR, Wellman RJ. A sensitization-homeostasis model of nicotine craving, withdrawal, and tolerance: integrating the clinical and basic science literature. Nicotine Tob Res. 2005;7(1):9-26.

18. Luoto R, Uutela A, Puska P. Occasional smoking increases total and cardiovascular mortality among men. Nicotine Tob Res. 2000;2(2):133-139. 
19. Garfinkel L, Stellman SD. Smoking and lung cancer in women: findings in a prospective study. Cancer Res. 1988;48(23):6951-6955.

20. Jiménez-Ruiz C, Kunze M, Fagerström KO. Nicotine replacement: a new approach to reducing tobacco-related harm. Eur Respir J. 1998;11(2): 473-479.

21. Benowitz NL. Cotinine as a biomarker of environmental tobacco smoke exposure. Epidemiol Rev. 1996;18(2):188-204.

22. Boffetta P, Clark S, Shen M, Gislefoss R, Peto R, Andersen A. Serum cotinine level as predictor of lung cancer risk. Cancer Epidemiol Biomarkers Prev. 2006;15(6):1184-1188.

23. Strachan DP, Jarvis MJ, Feyerabend C. The relationship of salivary cotinine to respiratory symptoms, spirometry, and exercise-induced bronchospasm in seven-year-old children. Am Rev Respir Dis. 1990;142(1): $147-151$.

24. Whincup PH, Gilg JA, Emberson JR, et al. Passive smoking and risk of coronary heart disease and stroke: prospective study with cotinine measurement. BMJ. 2004;329(7459):200-205.

25. Hecht SS, Carmella SG, Chen M, et al. Quantitation of urinary metabolites of a tobacco-specific lung carcinogen after smoking cessation. Cancer Res. 1999;59(3):590-596.

26. International Agency for Research on Cancer [IARC]. Smokeless Tobacco and Tobacco-specific Nitrosamines. Lyon: IARC; 2007. Available from: http://www.iarc.fr/en/publications/list/monographs/ index.php. Accessed September 19, 2012.

27. Goniewicz ML, Havel CM, Peng MW, et al. Elimination kinetics of the tobacco-specific biomarker and lung carcinogen 4-(methylnitrosamino)1-(3-pyridyl)-1-butanol. Cancer Epidemiol Biomarkers Prev. 2009;18(12):3421-3425.

28. Berg CJ, Nehl E, Sterling K, et al. The development and validation of a scale assessing individual schemas used in classifying a smoker: implications for research and practice. Nicotine Tob Res. 2011;13(12):1257-1265.
29. American College Health Association. American College Health Association: National College Health Assessment spring 2007 reference group data report (abridged). J Am Coll Health. 2008;56(5):469-479.

30. Centers for Disease Control and Prevention. Youth Risk Behavior Surveillance: National College Health Risk Behavior Survey - United States, 1995. MMWR CDC Surveill Summ. 1997;46(6):1-56. Available from: http://www.cdc.gov/mmwr/preview/mmwrhtmL/00049859.htm. Accessed July 7, 2006.

31. American College Health Association. American College Health Association-National College Health Assessment Spring 2008 Reference Group Data Report (abridged): the American College Health Association. J Am Coll Health. 2009;57(5):477-488.

32. Abuse and Mental Health Services Administration Substance. National Survey on Drug Use and Health, 2008: Codebook. SAMHSA, Office of Applied Studies, Rockville, Md, 2009, http://www.icpsr.umich.edu/ cgi-bin/file?comp $=$ none\&study $=26701 \& d s=1 \&$ file_id $=994670$

33. Fagerstrom KO, Schneider NG. Measuring nicotine dependence: a review of the Fagerstrom Tolerance Questionnaire. J Behav Med. 1989;12(2):159-182.

34. Jacob P 3rd, Havel C, Lee DH, Yu L, Eisner MD, Benowitz NL. Subpicogram per milliliter determination of the tobacco-specific carcinogen metabolite 4-(methylnitrosamino)-1-(3-pyridyl)-1-butanol in human urine using liquid chromatography-tandem mass spectrometry. Anal Chem. 2008;80(21):8115-8121.

35. Goniewicz ML, Eisner MD, Lazcano-Ponce E, et al. Comparison of urine cotinine and the tobacco-specific nitrosamine metabolite 4-(methylnitrosamino)-1-(3-pyridyl)-1-butanol (NNAL) and their ratio to discriminate active from passive smoking. Nicotine Tob Res. 2011;13(3):202-208.
Current Biomarker Findings

\section{Publish your work in this journal}

Current Biomarker Findings is an international, peer-reviewed, open access journal publishing original research, reports, reviews and commentaries on all areas of biomarker research. The manuscript management system is completely online and includes a very quick and fair

\section{Dovepress}

peer-review system. Visit http://www.dovepress.com/testimonials.php to read real quotes from published authors. 